\title{
CPOE in Non-Surgical Versus Surgical Specialties: A Qualitative Comparison of Clinical Contexts in the Medication Process
}

\author{
Zahra Niazkhani $^{*}{ }^{1,2}$, Habibollah Pirnejad ${ }^{1,2}$, Antoinette de Bont ${ }^{1}$ and Jos Aarts ${ }^{1}$ \\ ${ }^{I}$ Institute of Health Policy and Management (iBMG), Erasmus University Rotterdam, Rotterdam, The Netherlands \\ ${ }^{2}$ Health Information Technology (HIT) Group, Urmia University of Medical Sciences, Urmia, Iran
}

\begin{abstract}
Background: Computerized provider order entry (CPOE) systems are implemented in various clinical contexts of a hospital. To identify the role of the clinical context in CPOE use, we compared the impact of a CPOE system on the medication process in both non-surgical and surgical specialties.

Methods: We conducted a qualitative study of surgical and non-surgical specialties in a 1237-bed, academic hospital in the Netherlands. We interviewed the clinical end users of a computerized medication order entry system in both specialty types and analyzed the interview transcripts to elicit qualitative differences between the clinical contexts, clinicians' attitudes, and specialty-specific requirements.

Results: Our study showed that the differences in clinical contexts between non-surgical and surgical specialties resulted in a disparity between clinicians' requirements when using CPOE. Non-surgical specialties had a greater medication workload, greater and more diverse information needs to be supported in a timely manner by the system, and thus more intensive interaction with the CPOE system. In turn these factors collectively influenced the perceived impact of the CPOE system on the clinicians' practice. The non-surgical clinicians expressed less positive attitudes compared to the surgical clinicians, who perceived their interaction with the system to be less intensive and less problematic.

Conclusion: Our study shows that clinicians' different attitudes towards the system and the perceived impact of the system were largely grounded in the clinical context of the units. The study suggests that not merely the CPOE system, the technology itself, influences the perceptions of its users and workflow-related outcomes. The interplay between technology and clinical context of the implementation environment also matters. System design and redesigning efforts should take account of different units' specific requirements in their particular clinical contexts.
\end{abstract}

Keywords: Clinical context, CPOE, medication, medical order entry systems, surgical, non-surgical.

\section{BACKGROUND}

The successful deployment of computerized provider order entry (CPOE) systems requires a thorough understanding of the clinical workflow that these systems are intended to support [1]. Many diverse socio-technical factors in clinical workflow influence the deployment of CPOE systems $[2,3]$. One of these factors, as found in a recent systematic review, is the context of the clinical environment [4]. Ash et al. in a cross-site qualitative study pointed out the importance of context, including the clinical context of a unit in which CPOE systems are put to use [5].

Depending on the patients' needs, hospital care is delivered by various clinical specialties, each one having its own clinical context. The clinical context of a specialty consists of the interrelated conditions within which the examination and treatment of patients take place. The clinical profile of patients and their needs influences how this clinical context is shaped. The clinical context in turn influences both the way clinicians work and their work

\footnotetext{
*Address correspondence to this author at the Health Information Technology (HIT) Group, Urmia University of Medical Sciences, P.O. Box: 1138, Urmia, Iran; Tel: +98-441-2752305; Fax:+98-441-2770047;

E-mails: niazkhani@bmg.eur.nl or zahraniazkhani@yahoo.com
}

requirements. In order to determine the impact of the clinical context on CPOE use, Callen et al. compared the use of a computerized test management system between emergency departments and hematology wards [6,7]. The study showed that different clinical contexts highly impacted the clinicians' use of and attitudes towards CPOE. The research suggested that the contextual variation between clinical units should be taken into account when these systems are deployed. Nevertheless, only a limited number of studies have paid attention to this factor.

Many studies of workflow with CPOE systems include both surgical and non-surgical specialties in their evaluations (see for instance [8-10] ). In a review of the literature [1], we found only a limited number of studies that specifically compared the workflow-related outcomes with respect to the type of specialty [11-13]. Kaplan et al. found that nonsurgical physicians issued a larger rate of verbal orders than surgical physicians did after a CPOE system was in place [11]. Bates et al. evaluated the effect of computerized order entry on non-surgical and surgical house-staff time [12]. They found that although both groups spent more time on computerized order writing than on writing orders on paper, ordering with the CPOE system was more time-consuming for surgical staff than for non-surgical staff. They reported that the non-surgical staff recovered some of the time spent 
in activities that were accomplished more quickly after CPOE. Lee et al. studied the level of satisfaction with the same CPOE system and found that non-surgical clinicians were significantly more satisfied than the surgical clinicians [13].

Interestingly, all three studies used quantitative research methodology; however, none of the three evaluated why different outcomes between the specialties were seen. To answer this question, qualitative studies are known to be suitable methods [14]. Nevertheless, to our knowledge, no study to date has qualitatively compared the use of CPOE in the clinical context of non-surgical versus surgical specialties. No other study has identified the role-playing elements in their clinical contexts that affect the impact of CPOE, as well as the clinicians' use of and attitudes towards the system.

The objective of this study was thus to explore and understand which elements of a clinical context play a prominent role in the deployment of a CPOE system and how these elements affect workflow efficiency with the system. Understanding this issue, we believe, can help to identify and address the specialty-specific workflow requirements in design, implementation, and maintenance of CPOE systems in order to correspond better to existing needs. For this purpose, we conducted a qualitative study in which we compared the impact of a CPOE system on the medication process of two different clinical contexts in surgical and non-surgical specialties in a Dutch academic hospital.

\section{METHODS}

\subsection{Study Setting and the CPOE System}

This study was conducted at Erasmus University Medical Center, a 1237-bed academic hospital in Rotterdam, the Netherlands. This hospital has been using a vendor-based CPOE system, Medicatie/EVS ${ }^{\circledR}$ (Leiden, the Netherlands) in all inpatient settings except intensive care units (ICUs) since March 2005. The pharmacy department was responsible for both implementing the CPOE system and training its users in the hospital. As computerized physician order entry is mandatory in this hospital, physicians order electronically almost all medication orders for non-ICU, hospitalized patients. Since this hospital is a teaching hospital, the residents order the majority of the medication.

For medication order entry, a physician selects a drug and its dosage form, strength, administration route, dosage regimen, start date and time in Medicatie/EVS ${ }^{\circledR}$ (Fig. 1). The system generates safety alerts for drug-drug interactions, overdose, and duplicate orders. A detailed description of Medicatie/EVS ${ }^{\circledR}$ has been published elsewhere [15]. After physicians enter electronic orders, nurses receive medication orders printed on special labels called medication order (MO) labels. MO labels are then affixed to a Kardex card, which is the paper-based administration registration record. Supply of in-stock medication is controlled by the pharmacy technicians who scan in the ward stock two or three times per week. Whenever an MO label contains an out-of-stock drug, nurses can select the drug in the system and thereby send an electronic drug request to the pharmacy. The system's features are the same in all clinical wards. The details of the medication process, the MO labels, and the administration record have been described elsewhere [1618].

\subsection{Study Design and Data Collection}

The two types of non-surgical and surgical specialties were chosen for this study because firstly, the differences between their patients' clinical profiles and consequently between their clinical contexts are easily distinguishable. Secondly, in our prior study [17], we noticed some subtle but serious differences between these two types of specialties regarding the impact of CPOE on workflow that merited further evaluation. In this study we aimed at exploring the issue in greater detail, and therefore we collected additional data.

The first and the second authors conducted 25 interviews in total with physicians, nurses, and pharmacists between November 2006 and February 2009. During the interviews we reviewed and discussed the impact of the system on the interviewees' roles in the medication process, their medication-related tasks, their communication and collaboration with other care professionals, and, whenever appropriate, their specialty-specific requirements in the process.

For the purpose of the present study, we used 22 interviews with (16 non-surgical and six surgical) physicians and nurses. Table 1 provides details of their wards and professional status. The interviewees were among the respondents to our e-mail invitation sent to the key informant users and those recommended by the head of departments. Participation in the study was voluntary; no incentives were provided for participation. In each specialty type, we purposefully recruited the interviewees from both attending and resident groups. Similarly, we had both head nurses and practicing nurses in each specialty type. This diversity among interviewees helped us to get a better overview of various perspectives concerning the use of the system. It also helped us to get a deeper understanding of the existing clinical contexts. At the time of our study the interviewees had enough experience with the system to report on the process.

The interviews were in-depth, semi-structured, one-onone, and face-to-face and conducted in the interviewees' own clinical settings. This allowed us to observe how they were using the system and its printouts in the medication process. The field notes of these observations were also considered for analysis. All the interviews were voice-recorded.

\subsection{Data Analysis Process}

Interviews were transcribed verbatim and analyzed. The analysis sought to elicit inter-specialty differences as reported by clinicians. In this step, we adopted the approach explained by Ash and Guappone for data analysis about a topic that is more known: using "code lists or templates designed ahead of time" [19]. Drawing upon both the literature and our own experience in this study field, we used the following list of codes to analyze the transcripts:

- qualitative differences between non-surgical and surgical services in the clinical context of the 


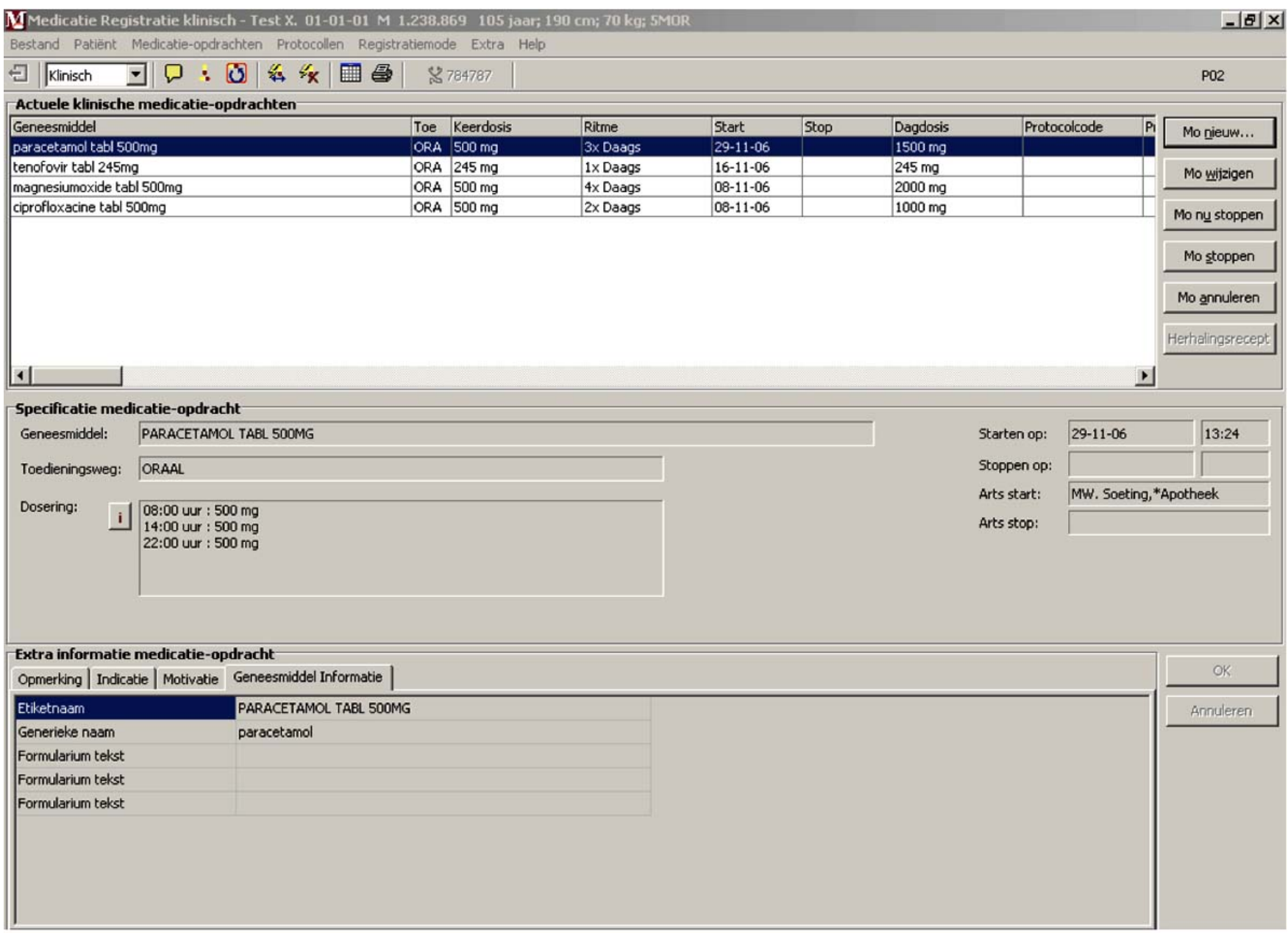

Fig. (1). A screen shot of the CPOE system for physicians

medication process which influence the use of a CPOE system,

- clinicians' attitudes towards CPOE in each specialty type.

- The specialty-specific requirements in the post-CPOE medication process.

For the analysis, the interview transcripts of non-surgical physicians were contrasted with those of surgeons. A similar analysis was conducted for the non-surgical versus surgical nurses.

\section{RESULTS}

The results are presented on the basis of the themes that emerged regarding the difference between the clinical contexts of surgical and non-surgical specialties. These themes were: 1) the clinical profile of patients and their medication orders, 2) information needs and communication patterns of clinicians, and 3) clinicians' attitudes towards the system.

\subsection{Clinical Profile of Patients and Their Medication Orders}

The clinical profiles of the patients admitted to each specialty greatly influenced the load of the medication work

Table 1. Wards and professional Status of the Interviewees in Each Specialty Type

\begin{tabular}{|c|c|c|}
\hline Specialty Type & Wards (\# of Interviews) & Interviewees' Professional Status \\
\hline \hline Non-surgical & $\begin{array}{c}\text { General internal medicine (two), gastroenterology (two), nephrology } \\
\text { (three), hematology (four), pulmonology (one), psychiatry (one), } \\
\text { metabolic diseases (one), and pediatrics (two) }\end{array}$ & $\begin{array}{c}\text { Six attending physicians, two residents, two head } \\
\text { nurses, and six practicing nurses }\end{array}$ \\
\hline Surgical & $\begin{array}{c}\text { General surgery (three), urology (one), neurosurgery (one), and pediatric } \\
\text { surgery (one) }\end{array}$ & $\begin{array}{c}\text { An attending surgeon, a surgery resident, a head nurse, } \\
\text { and three practicing nurses }\end{array}$ \\
\hline
\end{tabular}


and, consequently, the clinicians' interaction with the CPOE system. Patients in non-surgical units generally had several morbidities and 'medication' was one of the most important interventions to control their illness. Although the number of patients admitted to these two types of specialties was reported equally, the number of medications to start, stop, and change per patient was noticeably higher in the nonsurgical units. Non-surgical physicians referred to the workload especially related to newly admitted patients; occasionally they had to issue some 20 prescriptions for a single patient. The higher number of medication orders made them interact more with the system for structured order entry. For non-surgical nurses as well, procuring and administering these medications to patients was an extended process.

In contrast, care in the surgical units was mainly surgeryoriented. During interviews, surgeons emphasized the importance of surgery and that in their units, surgical care is far more predominant than medication work. They did not consider medication work as their 'core business' and pointed out that their patients did not generally use many medications, and if they did, these medications were mainly controlled by other physicians than surgeons during their hospital stay. For example, for patients who had undergone a kidney transplant, the medication work was predominantly conducted by nephrologists. In the case of pain management issues, anesthesiologists were involved. This meant that the number of medications started by surgeons would have been few. During the weekly ground rounds, which were performed together with other physicians, patients' medications were controlled by an internist. One of the surgeons referred to this as an 'automatic backup'. The system supported the surgeons in sharing medication information during their consultation with the internist.

The diversity of the medication orders was reported to be greater in non-surgical units than in surgical ones. The surgeons stated that three groups of medications (i.e., analgesics, antibiotics, and anti-thrombotic agents) comprised almost $80 \%$ of the medications used in surgical units. As most of these were normally available in these units' medication stocks nurses did not need to request them from the pharmacy. In contrast, nurses in non-surgical units reported normally having patients using a number of various medications not available in their unit's stocks, requiring them to put timely requests through the system to obtain these medications from the pharmacy.

\subsection{Information Needs and Communication Patterns of Clinicians}

As emphasized by our interviewees, higher numbers of medication changes generally followed the higher level of medication use in a unit. This is considered an indicator of a dynamic medication process. To meet this dynamic medication process required clinicians to have timely access to the patients' diverse and most up-to-date information for decision-making as well as for monitoring the effects. The system fell short of furnishing physicians with the full information required, especially in non-surgical wards, because on the one hand, the system was not available when decisions were being made at the patient's bedsides. And on the other hand, when orders were being entered in the physicians' offices, the medication administration records were not available to enable the monitoring the effects. This differed from the flexible and transportable paper-based system which had both medication records and information on the patient's clinical condition available at the bedsides. As a result, non-surgical physicians referred to the cognitive load of having to recall many patients' details from memory. One senior gastroenterologist noted:

"... We lost information in the system. If you
think of a drug fever in a patient, it will take
considerable amount of time and puzzling
before you get all the information together-
the temperature, changing doses, and
medication-, until you discover that a
patient's fever has to do with that particular
drug... If a patient is using several groups of
drugs and has several types of illnesses,
especially elderly patients and complicated
post-ICU patients, this becomes really
important".

As the load of medication orders was higher in nonsurgical units and ordering them by the system was timeconsuming, non-surgical physicians reported issuing many verbal orders. Based on a rough estimate by a non-surgical resident, 5-10 orders in each on-call shift of a resident were first communicated verbally. These verbal orders were entered into the system later on-even a few days later-by the initiating physicians or sometimes by their physician colleagues upon request by nurses.

Non-surgical clinicians were more concerned about the ability of the system to support their on-time communication with nurses than surgeons were. Access to the most updated medication information required non-surgical clinicians to communicate closely. While non-surgical physicians referred to the need to have nursing collected data integrated with their own, for surgeons the verbal communication of this information by nurses seemed sufficient. To integrate diverse information manually, non-surgical clinicians stressed the necessity of applying other methods of communication such as direct notification, phone calls, and co-existing paperbased orders besides the electronic orders. A hematology resident highlighted:

\section{"...[with this system] I don't think you can communicate effectively with the nurses, because you always have to tell them or write it down for them, I don't think these computers can replace that".}

However, the surgeons in our study thought that there was no need for additional communication of orders to nurses beyond the system. While at the time of our research, directly informing nurses about issuing the prescription labels was made mandatory by the implementation team, a surgery resident told us:
“...they (nurses) will just receive prescription labels ... then they look at the names and put them on the Kardex cards. So, we don't have to call them. But, I think it would be polite if you went along the system and told the nurses that you've put in an order for a drug for $\mathrm{Mr}$. A. It would be good but it isn't a must". 
Our interviews with nurses also revealed that the communication of orders by the system without verbal notification was considered less problematic by surgical nurses than by non-surgical nurses. This was because surgical nurses received fewer medication orders and if they did, those orders were mainly routine medications that they could manage even without being informed by the surgeons. A surgical nurse explained:

"... we know in some operations it is quite normal to have antibiotics. So when we see it [the MO label] we say, 'Yes, OK!' Because, most patients receive antibiotics before the operation starts".

The technical problems affecting access to information such as system downtime, printer problems, as well as the support shortcomings of the maintenance team were predominantly put forward by non-surgical rather than surgical clinicians. Non-surgical clinicians were concerned that any unavailability of this "vital system," as one senior attending put it, even for a short period, could hinder their workflow to a great degree. Usability issues, such as the suboptimal presentation of medication information on the computer screen and MO labels were discussed mainly by non-surgical physicians and nurses. It is noteworthy that surgical clinicians experienced these issues less frequently and perceived them as less of a hindrance to their work. Among non-surgical clinicians, however, these problems appeared to be more frustrating. One non-surgical clinician echoed this frustration when he said:

"The problem is that although people on the project team are very willing, what a doctor wants is just a running system. He is not an ICT person [who can] solve the implementation problems. It is not my job, I'm a doctor. It is not my problem if an ICT solution does not work, that is an ICT problem".

\subsection{Clinicians' Attitudes Towards the CPOE}

There were subtle differences between the two types of specialties in clinician's attitudes to CPOE. Non-surgical physicians sometimes expressed less positive attitudes towards the post-implementation medication process than surgical physicians did. In this regard, surgeons expressed more confidence in the CPOE system than non-surgical physicians did. The following quote from a surgery resident spells it out:

\section{"I am glad that Medicator [the name users have given to the CPOE system in this hospital] is here now. It's a very good system and a clear program. I don't see any problems [while working with the system] at the moment".}

However, non-surgical specialists viewed a structural difference between the pre- and post-implementation medication process, especially in the prescribing phase and also in communicating orders with nurses, which sometimes affected their workflow negatively. These specialists often mentioned that the supportive features of the system were somehow offset by the problems they were experiencing in the medication process. For most of them, unavailability of information at the time of decision-making, especially for complex patients as mentioned above, was among its biggest flaws. While comparing pre-implementation bedside orderwriting on paper to the electronic order entry in the physicians' offices, an attending physician explained:

"In theory, if you work in a very structured
way, you visit a patient and then you go to
your computer to order medications. The main
worry is on the busy on-call shifts with lots of
beeping, telephones, and calls from the
emergency room. So, it may well be the case
that the paper of notes [taken during bedside
visits] goes into the pocket of the physician
and he runs to the emergency department and
starts working there, and three hours later he
remembers that he has to do order entry on the
ward. Although he can access the system from
there, the worrying thing is that the doctor
who visited a patient is looking at, let's say,
potentially unreliable information and working
with a scrap of paper which gives him clues on
what to do and what to look for. That is not the
best a physician can offer to his patients".

There were perceptions among non-surgical specialists that the system served the pharmacy department's workflow more than it served the clinicians' workflow on the wards. One nephrology resident commented:

\section{"I think Medicator is more or less for the pharmacy [so that they can] see what to deliver; it's not really very good for the practitioners".}

Similarly, the lack of ownership in implementing this system was an issue among the non-surgical specialists. A non-surgical specialist commented:

"In my opinion, the system made a wrong
start, and that is because the system was
developed particularly by the pharmacists. I
can understand the initiatives led to starting
the project by the pharmacists, but as the
system was introduced it was a pharmacist's
system. For the pharmacists it makes things
more controllable and easier, although if you
look at the prescription process as a chain of
events there are many possible flaws in the
system".

Surgical nurses also seemed to be happier with the system than non-surgical nurses were. While non-surgical nurses referred to the problems especially at the beginning for both physicians and nurses, they reported that they were used to these problems because they "could not change the situation anyway". However, the surgical nurses thought that the CPOE system itself worked pretty well. Their main reason for that was the legible medication orders issued by the surgeons themselves. These nurses reported that the system freed them from preparing the list of medications to be signed by surgeons, as was sometimes the case before the implementation. 
Last but not least, the two groups of nurses also held different attitudes towards the sources of the problems causing disruption in their workflow. Similar to surgeons, surgical nurses expressed their confidence in the CPOE system; they thought the problems were caused by the way the users (i.e., surgeons, nurses, and the pharmacy staff) worked with the system and did not associate problems with the system itself. For example, a surgery head nurse stressed that after the implementation nurses perceived their workload to be highly dependent on whether the surgeons entered orders directly after medical rounds or did this later on. Another surgery nurse added:

\section{"You know, what I noticed is that it is not a priority for the physicians [surgeons]. They think it's the last thing to do. They don't actually know that we need them to order some medicine".}

Non-surgical nurses noted the complexity of the postimplementation medication process. These nurses mentioned that the interplay between users and the system-generated printouts demanded extra cognitive efforts as opposed to the paper-based system in which they had all a patient's medication orders on a single sheet. In spite of having legible orders, as majority noted, having to sort out the MO labels per patient resulted in the cognitive overload. A nonsurgical head nurse noted:

\begin{abstract}
"Every day lots of stickers [MO labels] come from Medicator. They [Nurses] have to put lots of stickers onto the Kardex cards. Sometimes the names of patients, [set in] very small [print], look alike; [then] sometimes one patient's stickers get put on the Kardex card of another patient. So tell me: Is it the nurse's fault or because it [the information] is [in] very small [print]?"
\end{abstract}

\section{DISCUSSION}

In this study we examined the role of the clinical context in CPOE use. We found that three main elements of the clinical contexts influenced the impact of a CPOE system, and the clinicians' use of and attitudes towards CPOE. The first element is whether the clinical process that a CPOE system is intended to support is one of the core activities of a unit. This element defines the load of physicians' and nurses' tasks that are related to a CPOE system. The second element is how information-intensive the process is. This affects the diversity of information needed by clinicians in a clinical process and the communication load among them for gaining access to the information and making sense of it. This element plays a role in determining how well a CPOE system fulfils the information need. The third element is how time-intensive and urgent are the tasks that a CPOE system is intended to support. This influences the need to synchronize the interrelated tasks in a clinical process through a CPOE system. The interplay between these three elements defines the workload of a clinical process in general and the load of clinicians' interaction with a CPOE system in particular.

Our study showed that the differences in clinical contexts between non-surgical and surgical specialties resulted in a disparity between clinicians' requirements when using
CPOE. For example, because of the greater use of medications in non-surgical specialties, non-surgical clinicians had a greater and more diverse information needs to be supported in a timely manner by the system. Furthermore, the greater medication workload made their interaction with the system more intensive. These factors in turn collectively influenced the perceived impact of the CPOE system on the clinicians' practice. As a result, the clinicians' different attitudes towards the medication process, the CPOE system, and the sources of problems were largely grounded in the clinical context of the units. In our case, the non-surgical clinicians generally expressed less positive attitudes compared to the surgical clinicians.

Our study underscores Callen et al.'s findings in that the difference between the two types of clinical contexts results in different clinicians' use of and attitudes towards a CPOE system [6]. In our study, non-surgical clinicians were heavy users of the CPOE system and their core business, the medication process, was greatly dependent on the efficient usability of the system and its round-the-clock operation. This group required diverse and timely access to the medication-related information for management of patients' medication plans. Their higher medication workload made them interact with the system further and, consequently, to experience and discover the system's usability problems to a greater extent. Experiencing problems, in turn, led the nonsurgical clinicians to complain more about the system. This finding differs from Lee et al.'s study in which non-surgical physicians were more satisfied than surgical physicians [13]. Lee and colleagues attributed their finding to the surgeons' lesser experience with their CPOE system at the time of the survey, the inefficient order entry process for medications such as anti-thrombotic agents and analgesics, and the more time surgeons spent on order entry process [12, 13]. However, the surgeons in our study found order entry quite efficient through this CPOE system; it enabled quick and easy order entry of medications used in surgical units. Although the overall structure of the medication process for surgical units looked similar to the non-surgical ones (described in detail elsewhere $[16,17]$ ), the surgical clinicians had considerably less interaction with the system. They had less difficulty, for example, with information access and communication, although similar issues afflicted them as well.

Verbal orders are considered as a risk for medication errors in hospitalized patients [20]. CPOE systems reduce the number of verbal orders [11, 21]. However, verbal communication of orders still exists after CPOE implementation [11]. Consistent with Kaplan et al.'s finding [11], non-surgical physicians in our study reported a similarly high rate of verbal orders. Kaplan and colleagues however did not explain why this was the case. Our qualitative study suggests that the higher rate of verbal orders seen in non-surgical specialties has to do with the greater workload of the medication process for them. Therefore, we argue that here again the three elements in the clinical context of non-surgical specialties, that is, the number of medication orders to enter, their communication load, and the urgency of their implementation, played a role in physicians' use of verbal orders. 
Our findings have important implications for CPOE implementation in the mixed clinical contexts of a hospital. Depending on which kinds of orders a CPOE system is intended to support, for example medication, laboratory, radiology or other ancillary orders, the units which will use these orders the most should be identified. Identifying the heavy unit-users of an order entry module is an important issue in the deployment of CPOE systems. This not only helps the mindful investment of time and budget by considering those specialties that would benefit most from the implementation, but it also helps to involve them early in a CPOE implementation project and accommodate their work requirements better after implementation. Such an approach can facilitate managing the change process from paper-based systems to electronic systems [22].

This study suggests that not merely the CPOE system, the technology itself, influences the perceptions of its users and workflow-related outcomes of the implementation. But, the interplay between technology and clinical context of the implementation environment also matters. The study also suggests that workflow support or the lack of it with a CPOE system is dependent on the clinical context in which it is being used. Therefore, the impact on one specialty's workflow and its clinicians' attitudes should not be taken for granted as an indication generalizable to the entire hospital. The voice and choice of each specialty group should be taken into account in implementing and redesigning CPOE systems. These implications are especially relevant in the context of commercial systems, especially because many institutions do not have the expertise to tailor less flexible vendor-provided systems to the differing needs of specialties. This also calls for vendors to take a more active role in implementing such systems and become team players [23].

The last but not least, we have reported on a successful implementation site - as defined by Ash et al. [24] - where more than 80 percent of orders are entered by physicians electronically. However, the efficient use of the system for different specialty types in such successful sites merits further attention. Hospitals should take potential problems in workflow, experienced in the context of the implementation and reported by heavy users, more seriously and invest in on-time and proper solutions. This in turn will benefit the safety of the process, as one of the motivations for implementing such information systems. In fact, as Aarts and Gorman state, safety requires "an approach that addresses the complex interactions between people, and their technologies in specific work environments" [25].

\subsection{Strengths and Weaknesses of the Study}

To our knowledge, no study to date has qualitatively compared workflow with a CPOE system in surgical and non-surgical services. Triangulation of data at the interviewers' level (i.e., two researchers) and interviewees' level (i.e., diverse clinicians and their professional status in each specialty type) is also one of strengths of our study. One advantage of the diversity at the clinician level was that while the attending physicians referred to the issues related to the implementation process, the residents mainly reported on the practice-oriented issues. This helped us to understand the effect of different factors on workflow. Moreover, rather than focusing on the many advantages that this system has brought to the work of clinicians in both specialty types, we mainly paid attention to identifying the specialty-specific pitfalls and their requirements in the medication process. We believe that this way of thinking about operating systems can help distinguish the interplay between different factors in practice and help redesign both the system and the care process in a more productive way. Our study should not be dismissed as merely non-surgical clinicians' dissatisfaction with the system.

There are weaknesses in our study. Most of our interviewees came from non-surgical units. We think this is mainly because the primary list of the key informant users that was used for inviting the participants included more non-surgical than surgical clinicians. Nonetheless, this imbalance might have affected our findings. Next, this paper detailed the differences between the two specialties in terms of the impact of CPOE on the work of clinical end-users. It should be noted that we did not study the impact of CPOE on the clinical outcomes of patient care in these specialties, which should be carefully studied in future.

\section{CONCLUSION}

Our findings reinforce the importance of the clinical context in CPOE deployment. This study shows different perceived effects in the same organizational processes with one system in different clinical contexts. This finding highlights the role of various socio-technical elements of a clinical process that affect the outcome of an information technology implementation.

Our study also points out that the heavy unit-users of a CPOE system should be distinguished and involved early in an implementation project. In order to integrate the function of a system with the context-oriented practices of care professionals, system redesigning efforts should focus on end-user's needs and concerns in their particular clinical contexts. The use of one CPOE system in different clinical contexts should be carefully evaluated in future studies. This will help to identify how well one system responds to the specialty-specific requirements of various units and what customizations will benefit these requirements.

\section{ACKNOWLEDGEMENTS}

We thank the study participants for their valuable time and insights. We also greatly acknowledge the members of the Iranian Academic Association of Medical Informatics (IRAAMI, http://site.iraami.org). Their discussion of the paper and their valuable comments helped us to improve the paper greatly.

\section{REFERENCES}

[1] Niazkhani Z, Pirnejad H, Berg M, Aarts J. The impact of computerized provider order entry systems on inpatient clinical workflow: a literature review. J Am Med Inform Assoc 2009; 16(4): 539-49.

[2] Berg M. Patient care information systems and health care work: a sociotechnical approach. Int J Med Inform 1999; 55: 87-101.

[3] Aarts J, Ash J, Berg M. Extending the understanding of computerized physician order entry: Implications for professional collaboration, workflow and quality of care. Int J Med Inform 2007; 76(Suppl 1): 4-13.

[4] Gruber D, Cummings GG, LeBlanc L, Smith DL. Factors influencing outcomes of clinical information systems 
implementation: a systematic review. Comput Inform Nurs 2009; 27(3): 151-63 quiz 164-5.

[5] Ash JS, Gorman PN, Lavelle M, et al. Perceptions of physician order entry: results of a cross-site qualitative study. Methods Inform Med 2003; 42(4): 313-23.

[6] Callen JL, Westbrook JI, Braithwaite J. The effect of physicians' long-term use of CPOE on their test management work practices. $\mathrm{J}$ Am Med Inform Assoc 2006; 13(6): 643-52.

[7] Callen JL, Braithwaite J, Westbrook JI. Contextual implementation model: a framework for assisting clinical information system implementations. J Am Med Inform Assoc 2008; 15(2): 255-62.

[8] Campbell EM, Guappone KP, Sittig DF, Dykstra RH, Ash JS. Computerized provider order entry adoption: implications for clinical workflow. J Gen Intern Med 2009; 24(1): 21-6.

[9] Beuscart-Zephir MC, Pelayo S, Anceaux F, Meaux JJ, Degroisse $\mathrm{M}$, Degoulet P. Impact of CPOE on doctor-nurse cooperation for the medication ordering and administration process. Int $\mathrm{J}$ Med Inform 2005; 74(7-8): 629-41.

[10] Ash JS, Gorman PN, Hersh WR, Lavelle M, Poulsen SB. Perceptions of house officers who use physician order entry. Proc AMIA Symp 1999: 471-5.

[11] Kaplan JM, Ancheta R, Jacobs BR. Inpatient verbal orders and the impact of computerized provider order entry. J Pediatr 2006; 149(4): 461-7.

[12] Bates DW, Boyle DL, Teich JM. Impact of computerized physician order entry on physician time. Proc Annu Symp Comput Appl Med Care 1994: 996.

[13] Lee F, Teich JM, Spurr CD, Bates DW. Implementation of physician order entry: user satisfaction and self-reported usage patterns. J Am Med Inform Assoc 1996; 3(1):42-55.

[14] Kaplan B, Shaw NT. Future directions in evaluation research: people, organizational, and social issues. Methods Inform Med 2004; 43(3): 215-31.
[15] Kalmeijer MD, Holtzer W, van Dongen R, Guchelaar HJ. Implementation of a computerized physician medication order entry system at the Academic Medical Centre in Amsterdam. Pharm World Sci 2003; 25(3): 88-93.

[16] Niazkhani Z, van der Sijs H, Pirnejad H, Redekop WK, Aarts J. Same system, different outcomes: comparing the transitions from two paper-based systems to the same computerized physician orde entry system. Int J Med Inform 2009; 78(3): 170-81.

[17] Niazkhani Z, Pirnejad H, van der Sijs H, de Bont A, Aarts J. Computerized provider order entry system - does it support the inter-professional medication process? Methods Inform Med 2010; 49(1): 20-7.

[18] Pirnejad H, Niazkhani Z, van der Sijs H, Berg M, Bal R. Impact of a computerized physician order entry system on nurse-physician collaboration in the medication process. Int J Med Inform 2008; 77(11): 735-44.

[19] Ash JS, Guappone KP. Qualitative evaluation of health information exchange efforts. J Biomed Inform 2007; 40(6 Suppl): S33-9.

[20] Pharmacopeia U. USP patient safety CAPSLink. October 2002; [Accessed July 7, 2009] available at: http://www.usp.org

[21] Zamora N, Carter M, Saull-McCaig S, Nguyen J. The benefits of the MOE/MAR implementation: a quantitative approach. Healthc Q 2006; 10 Spec No: 77-83, 6 .

[22] Lorenzi NM, Riley RT. Managing change: an overview. J Am Med Inform Assoc 2000; 7(2): 116-24.

[23] Koppel R, Localio AR, Cohen A, Strom BL. Neither panacea nor black box: responding to three Journal of Biomedical Informatics papers on computerized physician order entry systems. J Biomed Inform 2005; 38(4): 267-9.

[24] Ash JS, Gorman PN, Lavelle M, et al. A cross-site qualitative study of physician order entry. J Am Med Inform Assoc 2003; 10(2): 188-200.

[25] Aarts J, Gorman P. IT in health care: sociotechnical approaches "To Err is System". Int J Med Inform 2007; 76(Suppl 1): S1-3.

(C) Niazkhani et al.; Licensee Bentham Open.

This is an open access article licensed under the terms of the Creative Commons Attribution Non-Commercial License (http://creativecommons.org/licenses/by-nc /3.0/) which permits unrestricted, non-commercial use, distribution and reproduction in any medium, provided the work is properly cited. 